\title{
A unified Hamiltonian formalism of Jahn-Teller and pseudo-Jahn-Teller problems in axial
}

\section{symmetries}

\author{
James Brown, ${ }^{\dagger}$ Robert A. Lang, ${ }^{\ddagger}$, and Tao Zeng ${ }^{*, \dagger}$ \\ $\dagger$ Department of Chemistry, York University, Toronto, Ontario, M3J1P3, Canada \\ $\ddagger$ Department of Physical and Environmental Sciences, University of Toronto Scarborough, \\ Toronto, Ontario, M1C 1A4, Canada \\ IChemical Physics Theory Group, Department of Chemistry, University of Toronto, \\ Toronto, Ontario, M5S 3H6, Canada \\ E-mail: tzeng@yorku.ca
}

\begin{abstract}
A formalism for expansions of all Jahn-Teller and pseudo-Jahn-Teller Hamiltonian operators in all axial symmetries is presented. The formalism provides Hamiltonian expansions up to arbitrarily high order and including an arbitrary number of vibrational modes, which are of arbitrary types. It consists of three equations and two tables. The formalism is user-friendly since it can be used without understanding its derivation. An example of $E_{3}^{\prime \prime} \otimes e_{1}^{\prime}$ Jahn-Teller interaction of cycloheptatrienyl cation is used to demonstrate the correctness of the formalism. A Python program is developed to automate the generation of Hamiltonian expansions for all axial Jahn-Teller and pseodo-Jahn-Teller problems, , and interface the expansions to quantum dynamics simulation program. This is the first unified Hamiltonian formalism for axial JahnTeller and pseudo-Jahn-Teller problems. And it is the only one.
\end{abstract}




\section{INTRODUCTION}

In the mid-1930s, the idea of instability of nuclear configurations in degenerate electronic states was conceived by Landau, Teller, and Jahn. ${ }^{1}$ This idea was formulated by Jahn and Teller in $1937,{ }^{2}$ after whom the resultant theorem is named. According to this theorem: (1) nonlinear polyatomic systems with $\geq 3$-fold symmetry have orbital degeneracy; (2) degenerate electronic states arising from this orbital degeneracy have non-totally symmetric electron density; (3) the non-totally symmetric force the electrons impart on the nuclear framework lowers its symmetry, and consequently lifts the degeneracy. ${ }^{1,3,4}$ Twenty years later, Öpik and Pryce proposed that similar symmetry-lowering also occurs for a non-degenerate electronic state, if there is a significant interaction between the state and (an)other electronic state(s) along the distortion. ${ }^{5}$ This phenomenon is termed pseudo-Jahn-Teller (pJT) distortion. The Jahn-Teller (JT) and pJT interactions are the only driving forces for spontaneous symmetry breaking in polyatomic systems. ${ }^{6}$ Their consequences, termed JT and pJT effects, are commonly seen in spectroscopy, structural chemistry, solid-state physics, and optoelectronics. ${ }^{7-18}$

The JT and pJT interactions belong to the broader category of vibronic interactions, since they involve interactions between electronic and vibrational degrees of freedom. ${ }^{3,19}$ Naturally, accurate vibronic Hamiltonians for $\mathrm{JT} / \mathrm{pJT}$ problems are of critical importance to simulate and understand the associated JT/pJT effects. Usually, one such Hamiltonian operator is resolved in a set of electronic states within which the JT/pJT interactions occur, and the set of states are selected to be diabatic states. ${ }^{20-25}$ The diabatic states vary smoothly along nuclear structural change, so that their Hamiltonian matrix elements are differentiable functions of vibrational coordinates. The matrix elements can hence be expanded as polynomial series of vibrational coordinates. Overall, the selections of the electronic states, vibrational coordinates, and the orders of the expansions determine the accuracy of the Hamiltonian. An appropriate selection of coordinates, e.g., a Morse-type coordinate instead of a conventional normal mode coordinate, can significantly reduce the necessary order of expansions. Still, in general, higher order expansions give more accurate a JT/pJT Hamiltonian. 
Standard JT/pJT models ${ }^{3,4}$ with the expansions truncated at the second order have been know to be inadequate for systems with large amplitude vibrations. ${ }^{26-34}$ Mathematical formulas for high order expansions of JT/pJT Hamiltonians are strongly desired. This is not just true for theoreticians, but for experimentalists as well. ${ }^{32,35-38}$ Without the knowledge of high order expansion formulas, spectroscopists will have to fit their accurate vibronic spectra using inaccurate models. This is likely to impair the interpretation of the spectra. In the end, "there must be a rigorous connection between the experimentally measured, 'effective' molecular parameters and the calculated ones." 38 Pioneering derivations of the high-order JT/pJT Hamiltonians were performed for classic problems, such as $E \otimes e$ in $C_{3 v}$ symmetry, ${ }^{26,27} T_{2} \otimes t_{2}$ and $T_{2} \otimes e$ problems in $T_{d}$ symmetry. ${ }^{39,40}$ The derivations were quickly extended to problems with more than one set of vibrational modes and one electronic term, e.g., the $\left(A_{1}+E\right) \otimes\left(a_{2}+e\right)$ problem in $C_{3 v}$ symmetry. ${ }^{30}$ There were also recent attempts to derive high-order expansions for spin-orbit JT/pJT Hamiltonians. ${ }^{41,42}$

These studies were dedicated to specific cases and, despite the unprecedentedly high orders attained therein, the reported expansions were still of finite orders. These pioneering studies motivated us to derive JT/pJT expansion formulas that are of arbitrarily high orders and are as inclusive as for one class of symmetries, instead of one symmetry. So far, we have successfully derived arbitrarily high order expansion formulas for all bimodal JT/pJT problems in trigonal symmetries, ${ }^{43,44}$ tetragonal symmetries, ${ }^{45}$ cubic group symmetries, ${ }^{46,47}$ and spin-orbit JT/pJT problems in trigonal and tetragonal symmetries. ${ }^{48}$ These formalisms are inclusive, yet not enough. Problems with more than two sets of JT/pJT active vibrational modes and of higher axial symmetries, e.g., pentagonal (cyclopentadienyl ring ${ }^{49,50}$ ), hexagonal (benzene ${ }^{51,52}$ ), and in general $n$-gonal (nanotubes, nanohoops, and nanoloops of different sizes ${ }^{53-56}$ ), await to be addressed. "It is somewhat tedious to evaluate all possible cases of quadratic activity", this is a statement about deriving second order JT Hamiltonian for the cyclopentadienyl ring in an up-to-date excellent review article on vibronic coupling. ${ }^{38}$ Naturally, it would be more "tedious" and prone to mistakes if we use conventional methods 
to derive even higher order Hamiltonians. The necessity and urgency of a JT/pJT Hamiltonian formalism, which covers higher orders expansions and more axial symmetries, are unambiguously demonstrated. In this work, we make a larger stride forward to derive a unified JT/pJT Hamiltonian formalism that covers all problems in all axial symmetries with arbitrary numbers of all types of vibrational modes. The resultant more inclusive formalism turns out to adopt a simpler form than the previous ones for trigonal and tetragonal bimodal problems. ${ }^{43-45}$

In Section 2, we briefly overview axial symmetries and the conventional symbols of $\mathrm{JT} / \mathrm{pJT}$ problems in those symmetries. The mathematical symbols that are used in our derivations are also introduced. The derivation is presented in Section 3. The new formalism is incorporated in a Python program, VHEGEN2.0, which generates axial JT/pJT expansion formulas with minimal information of a problem. This program is introduced in Section 4. Section 5 concludes this work.

\section{SYMBOLS, SETTING, AND TERMINOLOGIES}

\subsection{A brief review of axial symmetries}

Axial symmetries mean those with one principal axis, i.e., those of $C_{n}, S_{2 n}, C_{n h}, C_{n v}, D_{n}$, $D_{n d}$, and $D_{n h}$ point groups. Throughout this work, $n$ is reserved to label the $n$-gonal principal axis. If necessary, $n_{o}$ and $n_{e}$ are used to indicate $n$ being odd and even, respectively. Eigenvalues of $\hat{C}_{n}$ operators $\left(\chi^{C_{n}}\right)$ are the $n$-th roots of unity: $e^{i \kappa \frac{2 \pi}{n}}$ with $\kappa=0, \pm 1, \pm 2, \cdots, \pm \operatorname{Int}\left(\frac{n_{o}}{2}\right)$ for $n_{o} ; \kappa=0, \pm 1, \pm 2, \cdots, \pm\left(\frac{n_{e}}{2}-1\right), \frac{n_{e}}{2}$ for $n_{e}$. Int () means taking the integer part of the quotient as its argument. $\kappa=0$ and $\frac{n_{e}}{2}$ correspond to eigenvalue $\chi^{C_{n}}=1$ and -1 , giving the non-degenerate $A$ - and $B$-type irreducible representations (IRREPs). $C_{n}$-eigenfunctions of these two IRREPs can always be chosen to be real-valued. The other $\chi^{C_{n}}$ 's are in complexconjugate pairs, as are their corresponding eigenfunctions which are inter-converted by the time-reversal (TR) operator $\hat{\mathcal{T}}$. Each pair of the TR-related $C_{n}$-eigenfunctions form an $E$ - 
type IRREP. Given a $\hat{\mathcal{T}}$-invariant Hamiltonian, an eigenfunction and its complex-conjugate must have the same energy. This is why the E-type eigenstates form degenerate pairs. The $E$-type IRREPs are labeled as $E_{k}$ for $k=1,2, \cdots, \operatorname{Int}\left(\frac{n_{o}}{2}\right)$ or $\frac{n_{e}}{2}-1$. For trigonal and tetragonal symmetries, only $k=1$ is allowed and the subscript is neglected in literature for the only $E$-type IRREP therein.

As the symmetry is increased from $C_{n}$ to $D_{n}$, the extra $\hat{C}_{2}^{\prime}$ operator around (one of) the $C_{2}$ axis(axes) perpendicular to $C_{n}$ further differentiate the $A$ and $B$ IRREPs to $A_{k}$ and $B_{k}$ IRREPs, with $k=1,2$ depending on whether their $\chi^{C_{2}}=1$ or -1 . $\hat{C}_{2}^{\prime}$ interchanges the two $E$ complex-valued components as $\hat{\mathcal{T}}$, and there is no further labeling for the $E$-type IRREPs in the $C_{n}$ to $D_{n}$ symmetry increase. $C_{n v}$ is isomorphic to $D_{n}$, with $C_{2}^{\prime}$ being replaced by $\sigma_{v} . D_{2 n d}$ is isomorphic to $D_{4 n}$, with $C_{n}$ being replaced by $S_{n}$. From $C_{n_{o}}$ symmetry to $C_{n_{o} h}$ symmetry, prime and double-prime are added to the $A$ and $E$-type IRREP symbols of $C_{n_{o}}$ to indicate their $\hat{\sigma}_{h}$-parities. For $C_{n_{e} h}$ symmetries, it is conventional to add $g$ and $u$ subscripts to label the $A, B$, and $E$-type IRREPs to indicate their $\hat{I}$-parities, instead of the prime and double-prime $\hat{\sigma}_{h}$-parities. Similarly, from $D_{n_{o}}$ to $D_{n_{o} h}$ symmetry, prime and double-prime are added to IRREP symbols; from $D_{n_{e}}$ to $D_{n_{e} h}$ symmetry, $g$ and $u$ subscripts are added. $D_{n_{o} d}$ is isomorphic to $D_{n_{o} h}$, with $\sigma_{h}$ being replaced by $I$. Correspondingly, the prime and double-prime in the $D_{n_{o} h}$ IRREP symbols are replaced by $g$ and $u$, correspondingly, in the $D_{n_{o} d}$ IRREPs. $S_{2 n}$ is isomorphic to $C_{2 n}$, with the proper principal axis being replaced by the improper principal axis. They share the same character table. Given the aforementioned isomorphisms, it is only necessary to consider $C_{n}, C_{n h}, D_{n}$, and $D_{n h}$ symmetries in the derivation below.

\subsection{Electronic states and vibrational modes in axial $\mathrm{JT} / \mathrm{pJT}$ in- teractions}

We follow the convention of using upper case IRREP symbols to label electronic states and lower case symbols for vibrational modes. Real-valued components of an $\left|E_{k}\right\rangle$ state are 
labeled as $\left|X_{k}\right\rangle$ and $\left|Y_{k}\right\rangle$. They are defined so that they transform under $\hat{C}_{n}$ as

$$
\hat{C}_{n}\left|X_{k}\right\rangle=\cos \frac{2 k \pi}{n}\left|X_{k}\right\rangle+\sin \frac{2 k \pi}{n}\left|Y_{k}\right\rangle ; \hat{C}_{n}|Y\rangle=-\sin \frac{2 k \pi}{n}\left|X_{k}\right\rangle+\cos \frac{2 k \pi}{n}\left|Y_{k}\right\rangle .
$$

The two real-valued components of an $e_{k}$ vibrational modes $\left(e_{k x}\right.$ and $\left.e_{k y}\right)$ follow the same transformation. The two Cartesian coordinates of an $e_{k}$ mode are labeled as $x_{k}$ and $y_{k}$. A function $f\left(x_{k}, y_{k}\right)$ under $\hat{C}_{n}$ have the coordinates transform in the same way as Eq. 1 . It is more convenient to use polar coordinates $\rho_{k}$ and $\phi_{k}\left(x_{k}=\rho_{k} \cos \phi_{k}, y_{k}=\rho_{k} \sin \phi_{k}\right)$ to describe the action of $\hat{C}_{n}: \hat{C}_{n} f\left(\rho_{k}, \phi_{k}\right)=f\left(\rho_{k}, \phi_{k}-\frac{2 k \pi}{n}\right)$. The real-valued $\left|X_{k}\right\rangle$ and $\left|Y_{k}\right\rangle$ component states are combined to form eigenstates of $\hat{C}_{n}$ :

$$
\left(\left|+_{k}\right\rangle\left|-_{k}\right\rangle\right)=\left(\left|X_{k}\right\rangle\left|Y_{k}\right\rangle\right) \frac{1}{\sqrt{2}}\left(\begin{array}{cc}
1 & 1 \\
i & -i
\end{array}\right) ; \hat{C}_{n}\left| \pm_{k}\right\rangle=e^{\mp i \frac{2 k \pi}{n}}\left| \pm_{k}\right\rangle .
$$

As the symmetry is increased from $C_{n}$ to $C_{n v}, D_{n}, D_{n d}$, and $D_{n h}$, the presence of the $C_{2}^{\prime}$ or $\sigma_{v}$ further define the $\left|E_{k}\right\rangle$ and $e_{k}$ components: $\left|X_{k}\right\rangle$ and $e_{k x}$ must be symmetric with respect to one $\hat{C}_{2}^{\prime}$ action in $D_{n}, D_{n d}$, and $D_{n h}$, while $\left|Y_{k}\right\rangle$ and $e_{k y}$ must be antisymmetric. The same definitions apply to $C_{n v}$ symmetry but with $\sigma_{h}$ being replaced by $\sigma_{v}$. With such definitions,

$$
\begin{gathered}
\hat{C}_{2}^{\prime} f\left(\rho_{k}, \phi_{k}\right)=f\left(\rho_{k},-\phi_{k}\right), \hat{\sigma}_{v} f\left(\rho_{k}, \phi_{k}\right)=f\left(\rho_{k},-\phi_{k}\right), \\
\hat{C}_{2}^{\prime}\left| \pm_{k}\right\rangle=\left|\mp_{k}\right\rangle=\left(\left| \pm_{k}\right\rangle\right)^{*} ; \hat{\sigma}_{v}\left| \pm_{k}\right\rangle=\left|\mp_{k}\right\rangle=\left(\left| \pm_{k}\right\rangle\right)^{*} .
\end{gathered}
$$

Clearly, for $\left| \pm_{k}\right\rangle, \hat{C}_{2}^{\prime}$ and $\hat{\sigma}_{v}$ have the same effect as $\hat{\mathcal{T}}$. Examples of the $E$-type states and e-type modes in pentagonal and hexagonal symmetries are given in Figure S.1 and S.2 in Supporting Information (SI).

$z$ and $w$ are reserved to label coordinates of $a$ - and $b$-type modes, respectively. Like for 
those modes, a subscript $k=1,2$ is attached to the coordinates to label their $\hat{C}_{2}^{\prime} / \hat{\sigma}_{v}$-parities.

$$
\begin{aligned}
& \hat{C}_{n} f\left(z_{k_{a}}, w_{k_{b}}\right)=f\left(z_{k_{a}},-w_{k_{b}}\right), \\
& \hat{C}_{2}^{\prime} f\left(z_{k_{a}}, w_{k_{b}}\right)=f\left((-1)^{\delta_{k_{a}, 2}} z_{k_{a}},(-1)^{\delta_{k_{b}, 2}} w_{k_{b}}\right),
\end{aligned}
$$

where the Kronecker delta is used to indicate the possible sign flipping of the coordinates. Since $\left[\hat{\sigma}_{h}, \hat{C}_{2}^{\prime}\right]=\left[\hat{\sigma}_{h}, \hat{\sigma}_{v}\right]=\left[\hat{\sigma}_{h}, \hat{C}_{n}\right]=0$ and the commutations hold when $\hat{\sigma}_{h}$ is replaced by $\hat{I}$, we can dress the vibrational modes, the vibrational coordinates, and the electronic states with the $\hat{\sigma}_{h} / \hat{I}$-parities independent of their transformations under $\hat{C}_{n}, \hat{C}_{2}^{\prime}$, and $\hat{\sigma}_{v}$. For $e_{k}$-type vibrational coordinates, it is only necessary to dress $\rho_{k}$ with the $\hat{\sigma}_{h} / \hat{I}$-parities, not $\phi_{k} \cdot p, q=g, u,{ }^{\prime},{ }^{\prime \prime}$ are used to indicate the parities.

$$
\begin{aligned}
\hat{\sigma}_{h} f\left(z_{k_{a}, p_{a}}, w_{k_{b}, p_{b}}, \rho_{k_{e}, p_{e}}, \phi_{k_{e}}\right) & =f\left((-1)^{\delta_{p_{a}, "}} z_{k_{a}, p_{a}},(-1)^{\delta_{p_{b}, "}} w_{k_{b}, p_{b}},(-1)^{\delta_{p_{e}, "}} \rho_{k_{e}, p_{e}}, \phi_{k_{e}}\right) \\
\hat{I} f\left(z_{k_{a}, p_{a}}, w_{k_{b}, p_{b}}, \rho_{k_{e}, p_{e}}, \phi_{k_{e}}\right) & =f\left((-1)^{\delta_{p_{a}, u}} z_{k_{a}, p_{a}},(-1)^{\delta_{p_{b}, u}} w_{k_{b}, p_{b}},(-1)^{\delta_{p_{e}, u}} \rho_{k_{e}, p_{e}}, \phi_{k_{e}}\right)
\end{aligned}
$$

For systems with axial symmetries, the JT interactions always occur within one $E_{k}$-type state. They are conventionally labeled as, e.g., $E_{k} \otimes(a+b+e+\cdots)$, with the modes that actively modify the electronic Hamiltonian matrix elements among the $E_{k}$ component states being placed in the parentheses. The JT interactions can be viewed as intra-term vibronic interactions, since the electronic states arise from the same electronic term symbol. Intraterm interactions also include $A \otimes$ - and $B \otimes$-type problems. The pJT interactions occur between states of different energies. They are conventionally labeled as, e.g., $\left(E_{k}+A_{l}\right) \otimes$ $(a+b+e+\cdots)$. The vibrational modes included in the second parentheses actively modify the electronic Hamiltonian matrix elements between the states of two term symbols in the first parentheses. The pJT interactions are typical inter-term vibronic interactions. In both JT and pJT interactions, the VIBrational-coordinates-dependence of the electRONIC Hamiltonian matrix elements coins the term of vibronic interaction. 


\subsection{Fundamental expansion formula}

Given a problem with $N_{a} a$-type modes, $N_{b} b$-type modes, and $N_{e} e$-type modes, a monomial of the product of powers of their coordinates take the form of

$$
\left(\prod_{I_{a}}^{N_{a}} z_{k_{I_{a}}, p_{I_{a}}}^{M_{I_{a}}} \prod_{I_{b}}^{N_{b}} w_{k_{I_{b}}, p_{I_{b}}}^{M_{I_{b}}} \prod_{I_{e}}^{N_{e}} \rho_{k_{I_{e}}, p_{I_{e}}}^{\left|M_{I_{e}}\right|+2 K_{I_{e}}}\right) e^{i\left(\sum_{I_{e}}^{N_{e}} M_{I_{e}} \phi_{k_{I_{e}}}\right)} .
$$

$M_{I_{a}}$ etc. indicate the powers of the coordinates. Throughout this work, when indices are given in absolute value symbol, they can take all integer values. Otherwise, they can only take non-negative integer values. The $\left|M_{I_{e}}\right|+2 K_{I_{e}}$ power of $\rho_{k_{I_{e}}, p_{I_{e}}}$ guarantees no singularity at the $\rho_{k_{I_{e}}, p_{I_{e}}}=0$. The $\left.e^{i\left(\sum_{I_{e}}^{N_{e}} M_{I_{e}} \phi_{k_{I_{e}}}\right.}\right)$ phase factor with the integral $\left\{M_{I_{e}}\right\}$ is a term in the Fourier series of all $\left\{\phi_{k}\right\}$, which guarantees invariance with respect to $\hat{C}_{1}$ rotation. This invariance is a fundamental requirement for any matrix elements of JT/pJT Hamiltonians. Eq. 8 is applicable for both $n_{e^{-}}$and $n_{o^{-}}$gonal symmetries; we just need to set $N_{b}=0$ for

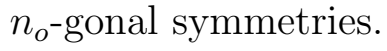

The ranges of $\left\{M_{I_{a}}\right\},\left\{M_{I_{b}}\right\},\left\{M_{I_{e}}\right\}$, and $\left\{K_{I_{e}}\right\}$ that satisfy $\sum_{I_{a}}^{N_{a}} M_{I_{a}}+\sum_{I_{a}}^{N_{b}} M_{I_{b}}+$ $\sum_{I_{e}}^{N_{e}}\left|M_{I_{e}}\right|+2 K_{I_{e}} \leq N_{\text {order }}$ give us all monomials of the coordinates that make the expansion up to $N_{\text {order }}$-th order. The expansion takes the form of

$$
C_{\left\{M_{I_{a}}\right\},\left\{M_{I_{b}}\right\},\left\{M_{I_{e}}\right\},\left\{K_{I_{e}}\right\}}\left(\prod_{I_{a}}^{N_{a}} z_{k_{I_{a}}, p_{I_{a}}}^{M_{I_{a}}} \prod_{I_{b}}^{N_{b}} w_{k_{I_{b}}, p_{I_{b}}}^{M_{I_{b}}} \prod_{I_{e}}^{N_{e}} \rho_{k_{I_{e}}, p_{I_{e}}}^{\left|M_{I_{e}}\right|+2 K_{I_{e}}}\right) e^{i\left(\sum_{I_{e}}^{N_{e}} M_{I_{e}} \phi_{k_{I_{e}}}\right)} .
$$

The Einstein convention of summing over duplicate indices is followed in all expansion formulas in this work. Specifically, the duplicate indices in Eq. 9 are $\left\{M_{I_{a}}\right\},\left\{M_{I_{b}}\right\},\left\{M_{I_{e}}\right\}$, and $\left\{K_{I_{e}}\right\} . N_{\text {order }}$ is not specified as this fundamental expansion formula is applicable for all orders. The $C_{\left\{M_{I_{a}}\right\},\left\{M_{I_{b}}\right\},\left\{M_{I_{e}}\right\},\left\{K_{I_{e}}\right\}}$ coefficient is in general complex-valued. It should be emphasized that Eq. 9 is a complete polynomial expansion formula. 


\section{Derivations}

The derivations of the expansion formulas for JT/pJT Hamiltonians are separated into two parts, electronic and vibrational. The electronic part results in symmetry-eigenvalues of electronic Hamiltonian matrix elements. The vibrational part results in expansion formulas that feature those eigenvalues. Those expansions are modules to be selected based on the needs of the electronic part. Such a modularized approach is of critical importance, as it makes possible to derive formalisms for thousands of problems in one work. ${ }^{44-47}$ As shown below, many of the matrix elements share the same set of symmetry-eigenvalues.

\subsection{Electronic part}

We start the derivation for the $E_{k}$-type JT problem. The Hamiltonian is resolved in the degenerate set $\left| \pm_{k}\right\rangle$ :

$$
\hat{H}=\left(\left|+_{k}\right\rangle\left\langle+_{k}|+|-_{k}\right\rangle\left\langle-_{k}\right|\right) H_{+_{k}+_{k}}^{r}+\left|+_{k}\right\rangle\left\langle-_{k}\right| H_{+_{k}-k}+\text { h.c. }
$$

The $r$ superscript indicates that $H_{+_{k}+_{k}}^{r}$ is a real-valued element, since it is a diagonal element. h.c. means the Hermitian conjugate of the explicitly written part with complex-valued elements. In this expression, h.c. only consists of one term, $\left|-_{k}\right\rangle\left\langle+_{k}\right| H_{+_{k}-{ }_{k}}^{*}$. The $\left|+_{k}\right\rangle\left\langle+_{k}\right|$ and $\left|-{ }_{k}\right\rangle\left\langle-{ }_{k}\right|$ dyads share the same matrix element as a consequence of TR-symmetry. The use of the $\hat{C}_{n}$-eigenstates $\left| \pm_{k}\right\rangle$ simplifies the expression of $\hat{H}$ under the action of $\hat{C}_{n}$ :

$$
\hat{C}_{n} \hat{H} \hat{C}_{n}^{-1}=\left(\left|+_{k}\right\rangle\left\langle+_{k}|+|-_{k}\right\rangle\left\langle-_{k}\right|\right) \hat{C}_{n} H_{+_{k}+_{k}}^{r}+e^{-i \frac{4 k \pi}{n}}\left|+_{k}\right\rangle\left\langle-_{k}\right| \hat{C}_{n} H_{+_{k}-{ }_{k}}+\text { h.c. }
$$

The vibronic Hamiltonian needs to be invariant under all symmetry operations of the relevant point group. In order to be $\hat{C}_{n}$-invariant, the two matrix elements need to satisfy

$$
\hat{C}_{n} H_{+_{k}+{ }_{k}}^{r}=H_{+_{k}+{ }_{k}}^{r} ; \hat{C}_{n} H_{+_{k}-k}=e^{i \frac{4 k \pi}{n}} H_{+_{k}-k}
$$


They are $\hat{C}_{n}$-eigenfunctions with the respective $\chi^{C_{n}} \mathrm{~s}$.

As the symmetry is increased to $D_{n}, \hat{H}$ needs to be invariant with respect to $\hat{C}_{2}^{\prime}$.

$$
\hat{C}_{2}^{\prime} \hat{H} \hat{C}_{2}^{\prime-1}=\left(\left|-{ }_{k}\right\rangle\left\langle-_{k}|+|+_{k}\right\rangle\left\langle+_{k}\right|\right) \hat{C}_{2}^{\prime} H_{+_{k}+_{k}}^{r}+\left|-_{k}\right\rangle\left\langle+_{k}\right| \hat{C}_{2}^{\prime} H_{+_{k}-k}+\text { h.c. }
$$

To have $\hat{C}_{2}^{\prime} \hat{H} \hat{C}_{2}^{\prime-1}=\hat{H}$, the two elements need to satisfy

$$
\hat{C}_{2}^{\prime} H_{+_{k}+k}^{r}=H_{+_{k}+k}^{r} ; \hat{C}_{2}^{\prime} H_{+_{k}-k}=H_{+_{k}-{ }_{k}}^{*}
$$

While $H_{+_{k}+_{k}}^{r}$ is a $\hat{C}_{2}^{\prime}$-eigenfunction with $\chi^{C_{2}^{\prime}}=1, H_{+_{k}-{ }_{k}}$ is not an eigenfunction, as the real and imaginary parts of $H_{+_{k}-k}$ feature different eigenvalues $\left(\chi_{R e}^{C_{2}^{\prime}}, \chi_{I m}^{C_{2}^{\prime}}\right)=(1,-1)$, respectively. We use $\left(\chi_{R e}^{C_{2}^{\prime}}, \chi_{I m}^{C_{2}^{\prime}}\right)$ to indicate the $\chi^{C_{2}^{\prime}}$-eigenvalues of the real and imaginary parts of a matrix element. $\left(\chi_{R e}^{C_{2}^{\prime}}, \chi_{I m}^{C_{2}^{\prime}}\right)=(1,0)$ for $H_{+_{k}+_{k}}^{r}$. The 0 indicates that the matrix element is real-valued.

As the symmetry is increased from $C_{n}$ to $C_{n h}$, all the ket-bra dyads are $\hat{\sigma}_{h}$-invariant (and $\hat{I}$-invariant if $n$ is even). Consequently, $\chi^{\sigma_{h} / I}=1$ for the two elements. The higher $D_{n h}$ symmetry is a composites of $D_{n}$ and $C_{n h}$ symmetries. The elements then need to carry all their symmetry-eigenvalues above. The unique matrix elements and their symmetryeigenvalues are summarized in the $E_{k, p}$ row of Table 1.

We then move on to the $\left(E_{k}+E_{l}\right)$-type pJT problem, whose Hamiltonian reads

$$
\hat{H}=\left(\left|+_{k}\right\rangle\left\langle+_{l}|+|-_{l}\right\rangle\left\langle-_{k}\right|\right) H_{+_{k}+l}+\left(\left|+_{k}\right\rangle\left\langle-_{l}|+|+_{l}\right\rangle\left\langle-_{k}\right|\right) H_{+_{k}-l}+\text { h.c. }
$$

Again, the dyads in each set of parentheses share the same matrix element as a consequence of TR-symmetry.

$$
\begin{aligned}
\hat{C}_{n} \hat{H} \hat{C}_{n}^{-1}= & e^{-i \frac{2(k-l) \pi}{n}}\left(\left|+{ }_{k}\right\rangle\left\langle+_{l}|+|-_{l}\right\rangle\left\langle-_{k}\right|\right) \hat{C}_{n} H_{+_{k}+_{l}} \\
& +e^{-i \frac{2(k+l) \pi}{n}}\left(\left|+{ }_{k}\right\rangle\left\langle-_{l}|+|+{ }_{l}\right\rangle\left\langle-_{k}\right|\right) \hat{C}_{n} H_{+_{k}-l}+\text { h.c. }
\end{aligned}
$$


Table 1: Forms of Hamiltonians of all intra- and inter-term vibronic Hamiltonians in axial symmetries, and the symmetry-eigenvalues of their matrix elements. Each set of the symmetry-eigenvalues are given for the matrix element in the same row.

\begin{tabular}{|c|c|c|}
\hline Problem & $\hat{H}$ & $\chi^{C_{n} / S_{n}},\left(\chi_{\operatorname{Re}}^{C_{2}^{\prime} / \sigma_{v}}, \chi_{\operatorname{Im}}^{C_{2}^{\prime} / \sigma_{v}}\right), \chi^{\sigma_{h} / I}$ \\
\hline$E_{k, p}$ & $\begin{array}{l}\left(\left|+_{k, p}\right\rangle\left\langle+_{k, p}|+|--_{k, p}\right\rangle\left\langle-{ }_{k, p}\right|\right) H_{+k, p}^{r}+_{k, p} \\
+\left|+_{k, p}\right\rangle\langle-k, p| H_{+_{k, p}-k, p}+\text { h.c. }\end{array}$ & $\begin{array}{c}1,(1,0), 1 \\
e^{i \frac{k k \pi}{n}},(1,-1), 1\end{array}$ \\
\hline$A_{k, p}$ & $\left|A_{k, p}\right\rangle\left\langle A_{k, p}\right| H_{A_{k, p}}^{r} A_{k, p}$ & $1,(1,0), 1$ \\
\hline$B_{k, p}$ & $\left|B_{k, p}\right\rangle\left\langle B_{k, p}\right| H_{B_{k, p} B_{k, p}}^{r}$ & $1,(1,0), 1$ \\
\hline$\left(E_{k, p}+E_{l, q}\right)$ & $\begin{array}{l}\left(\left|+_{k, p}\right\rangle\left\langle+_{l, q}|+|-{ }_{l, q}\right\rangle\left\langle-{ }_{k, p}\right|\right) H_{+_{k, p}+l, q} \\
+\left(\left|+_{k, p}\right\rangle\left\langle-l, q|+|+_{l, q}\right\rangle\langle-k, p|\right) H_{+_{k, p}-l, q}+\text { h.c. }\end{array}$ & $\begin{array}{l}e^{i \frac{2(k-l) \pi}{n}},(1,-1),(-1)^{\delta_{p, q}+1} \\
e^{i \frac{2(k+l) \pi}{n}},(1,-1),(-1)^{\delta_{p, q}+1}\end{array}$ \\
\hline$\left(E_{k, p}+A_{l, q}\right)$ & $\left(\left|+_{k, p}\right\rangle\left\langle A_{l, q}|+| A_{l, q}\right\rangle\left\langle-_{k, p}\right|\right) H_{+k, p} A_{l, q}+$ h.c. & $e^{i \frac{2 k \pi}{n}},\left((-1)^{\delta_{l, 2}},(-1)^{\delta_{l, 1}}\right),(-1)^{\delta_{p, q}+1}$ \\
\hline$\left(E_{k, p}+B_{l, q}\right)$ & $\left(\left|+_{k, p}\right\rangle\left\langle B_{l, q}|+| B_{l, q}\right\rangle\left\langle-_{k, p}\right|\right) H_{+k, p} B_{l, q}+$ h.c. & $e^{i \frac{(2 k-n) \pi}{n}},\left((-1)^{\delta_{l, 2}},(-1)^{\delta_{l, 1}}\right),(-1)^{\delta_{p, q}+1}$ \\
\hline$\left(A_{k, p}+A_{l, q}\right)$ & $\left(\left|A_{k, p}\right\rangle\left\langle A_{l, q}|+| A_{l, q}\right\rangle\left\langle A_{k, p}\right|\right) H_{A_{k, p} A_{l, q}}^{r}$ & $1,\left((-1)^{\delta_{k l}+1}, 0\right),(-1)^{\delta_{p, q}+1}$ \\
\hline$\left(B_{k, p}+B_{l, q}\right)$ & $\left(\left|B_{k, p}\right\rangle\left\langle B_{l, q}|+| B_{l, q}\right\rangle\left\langle B_{k, p}\right|\right) H_{B_{k, p} B_{l, q}}^{r}$ & $1,\left((-1)^{\delta_{k l}+1}, 0\right),(-1)^{\delta_{p, q}+1}$ \\
\hline$\left(A_{k, p}+B_{l, q}\right)$ & $\left(\left|A_{k, p}\right\rangle\left\langle B_{l, q}|+| B_{l, q}\right\rangle\left\langle A_{k, p}\right|\right) H_{A_{k, p} B_{l, q}}^{r}$ & $-1,\left((-1)^{\delta_{k l}+1}, 0\right),(-1)^{\delta_{p, q}+1}$ \\
\hline
\end{tabular}


The conditions for $\hat{C}_{n} \hat{H} \hat{C}_{n}^{-1}=\hat{H}$ are

$$
\hat{C}_{n} H_{+_{k}+l}=e^{i \frac{2(k-l) \pi}{n}} H_{+_{k}+l} ; \hat{C}_{n} H_{+_{k}-l}=e^{i \frac{2(k+l) \pi}{n}} H_{+_{k}-{ }_{k}} .
$$

In $D_{n}$ symmetry,

$$
\begin{aligned}
\hat{C}_{2}^{\prime} \hat{H} \hat{C}_{2}^{\prime-1}= & \left(\left|-{ }_{k}\right\rangle\left\langle-{ }_{l}|+|+{ }_{l}\right\rangle\left\langle+_{k}\right|\right) \hat{C}_{2}^{\prime} H_{+_{k}+_{l}} \\
& +\left(\left|-{ }_{k}\right\rangle\left\langle+_{l}|+|-{ }_{l}\right\rangle\left\langle+_{k}\right|\right) \hat{C}_{2}^{\prime} H_{+_{k}-l}+\text { h.c. }
\end{aligned}
$$

$\hat{C}_{2}^{\prime} \hat{H} \hat{C}_{2}^{\prime-1}=\hat{H}$ requires

$$
\hat{C}_{2}^{\prime} H_{+_{k}+l}=H_{+_{k}+l}^{*} ; \hat{C}_{2}^{\prime} H_{+_{k}-l}=H_{+_{k}-k}^{*}
$$

In $C_{n h}$ symmetry, to the $\hat{\sigma}_{h} / \hat{I}$-parities of the matrix elements must be equal to the parities

of the associated dyads (i.e., $(-1)^{\delta_{p, q}+1}$ ) to make the Hamiltonian $\hat{\sigma}_{h} / \hat{I}$-invariant. All these symmetry-eigenvalues of the $\left(E_{k}+E_{l}\right)$-type pJT problem are summarized in the $\left(E_{k}+E_{l}\right)$ row of Table 1. The derivations for the electronic parts of the other intra- and inter-term problems follow the same route and are presented in Section S.2 in SI. The forms of Hamiltonians of all vibronic problems in axial symmetries and the symmetry-eigenvalues of their matrix elements are summarized in Table 1.

\subsection{Vibrational part}

The symmetry-eigenvalues in Table 1 impose constraints onto the most general polynomial expansion formula of vibrational coordinates in Eq. 9. Some of the terms must be excluded and the coefficients must adopt specific forms to give the appropriate symmetry-eigenvalues. 
The monomial in Eq. 8 transforms under $\hat{C}_{n}$ as

$$
\begin{aligned}
& \left.\hat{C}_{n}\left(\prod_{I_{a}}^{N_{a}} z_{k_{I_{a}}, p_{I_{a}}}^{M_{I_{a}}} \prod_{I_{b}}^{N_{b}} w_{k_{I_{b}}, p_{I_{b}}}^{M_{I_{b}}} \prod_{I_{e}}^{N_{e}} \rho_{k_{I_{e}}, p_{I_{e}}}^{\left|M_{I_{e}}\right|+2 K_{I_{e}}}\right) e^{i\left(\sum_{I_{e}}^{N_{e}} M_{I_{e}} \phi_{k_{I_{e}}}\right.}\right) \\
& =\left(\prod_{I_{a}}^{N_{a}} z_{k_{I_{a}}, p_{I_{a}}}^{M_{I_{a}}} \prod_{I_{b}}^{N_{b}}(-1)^{M_{I_{b}}} w_{k_{I_{b}}, p_{I_{b}}}^{M_{I_{b}}} \prod_{I_{e}}^{N_{e}} \rho_{k_{I_{e}}, p_{I_{e}}}^{\left|M_{I_{e}}\right|+2 K_{I_{e}}}\right) e^{i\left(\sum_{I_{e}}^{N_{e}} M_{I_{e}}\left(\phi_{k_{I_{e}}}-\frac{2 k_{I_{e}} \pi}{n}\right)\right)} \\
& =\left(\prod_{I_{a}}^{N_{a}} z_{k_{I_{a}}, p_{I_{a}}}^{M_{I_{a}}} \prod_{I_{b}}^{N_{b}} w_{k_{I_{b}}, p_{I_{b}}}^{M_{I_{b}}} \prod_{I_{e}}^{N_{e}} \rho_{k_{I_{e}}, p_{I_{e}}}^{\left|M_{I_{e}}\right|+2 K_{I_{e}}}\right) e^{i\left(\sum_{I_{e}}^{N_{e}} M_{I_{e}} \phi_{k_{I_{e}}}\right)} e^{-i\left(\sum_{I_{e}}^{N_{e}} M_{I_{e}} k_{I_{e}}+\frac{n}{2} \sum_{I_{b}}^{N_{b}} M_{I_{b}}\right) \frac{2 \pi}{n}}
\end{aligned}
$$

The reason of using such monomials in the expansion is now obvious: they $\operatorname{are} \hat{C}_{n}$-eigenfunctions. To have $\chi^{C_{n}}=e^{i \frac{2 \kappa \pi}{n}}$, the power indices of the $e_{k}$-type and $b$-type coordinates must satisfy

$$
\sum_{I_{e}}^{N_{e}} M_{I_{e}} k_{I_{e}}+\frac{n}{2} \sum_{I_{b}}^{N_{b}} M_{I_{b}}=L n-\kappa
$$

where $L$ can take any integer values from $-\infty$ to $\infty$. Clearly, in Eq. 9 , only the $C_{\left\{M_{I_{a}}\right\},\left\{M_{I_{b}}\right\},\left\{M_{I_{e}}\right\},\left\{K_{I_{e}}\right\}}$ coefficients with their $\left\{M_{I_{b}}\right\}$ and $\left\{M_{I_{e}}\right\}$ indices satisfying Eq. 21 can be nonzero. We can then obtain expansions for the matrix elements in Table 1 according to their $\chi^{C_{n}}$ values. The $L n$ factor in Eq. 21 determines that there are in general fewer terms in the vibronic Hamiltonian expansions for systems with a higher axial symmetry. Generally, fewer combinations of indices on the left hand side of the equation match a larger $n$ periodicity on the right hand side.

The situations of $\kappa=0$ and $\frac{n_{e}}{2}\left(\chi^{C_{n}}=1,-1\right)$ are worth further discussion. $\hat{C}_{n^{-}}$ eigenfunctions with those $\chi^{C_{n}}$ values transform as $A$ - or $B$-type IRREPs and can be expanded using real-valued bases. We then choose the cos and sin functions of the phase angles in Eq. 9 to expand those $\hat{C}_{n}$-eigenfunctions:

$$
\begin{aligned}
& C_{\left\{M_{I_{a}}\right\},\left\{M_{I_{b}}\right\},\left\{\bar{M}_{I_{e}}\right\},\left\{K_{I_{e}}\right\}}^{c}\left(\prod_{I_{a}}^{N_{a}} z_{k_{I_{a}}, p_{I_{a}}}^{M_{I_{a}}} \prod_{I_{b}}^{N_{b}} w_{k_{I_{b}}, p_{I_{b}}}^{M_{I_{b}}} \prod_{I_{e}}^{N_{e}} \rho_{k_{I_{e}}, p_{I_{e}}}^{\left|M_{I_{e}}\right|+2 K_{I_{e}}}\right) \cos \left(\sum_{I_{e}}^{N_{e}} M_{I_{e}} \phi_{k_{I_{e}}}\right) \\
&+C_{\left\{M_{I_{a}}\right\},\left\{M_{I_{b}}\right\},\left\{\bar{M}_{I_{e}}\right\},\left\{K_{I_{e}}\right\}}^{s}\left(\prod_{I_{a}}^{N_{a}} z_{k_{I_{a}}, p_{I_{a}}}^{M_{I_{a}}} \prod_{I_{b}}^{N_{b}} w_{k_{I_{b}}, p_{I_{b}}}^{M_{I_{b}}} \prod_{I_{e}}^{N_{e}} \rho_{k_{I_{e}}, p_{I_{e}}}^{\left|M_{I_{e}}\right|+2 K_{I_{e}}}\right) \sin \left(\sum_{I_{e}}^{N_{e}} M_{I_{e}} \phi_{k_{I_{e}}}\right) .
\end{aligned}
$$


To avoid duplication of the cos and sin functions, one of the $\left\{M_{I_{e}}\right\}$ indices is restricted to take only nonnegative integer values. It is naturally to choose the first $e_{k}$-type mode to impose such an additional restriction, which is denoted by an overhead bar in $\left\{\bar{M}_{I_{e}}\right\}$ in the expression. The $C^{c}$ and $C^{s}$ coefficients are in general complex-valued. It is convenient to use Eq. 22 to derive the expansions with appropriate $\chi^{C_{2}^{\prime}}$. Note that Eq. 22 is just a special form of Eq. 9, and the constraint of Eq. 21 holds in Eq. 22 for $\kappa=0, \frac{n_{e}}{2}$.

Under the action of $\hat{C}_{2}^{\prime}$, the expansion in Eq. 22 becomes

$$
\begin{aligned}
& C_{\left\{M_{I_{a}}\right\},\left\{M_{I_{b}}\right\},\left\{\bar{M}_{I_{e}}\right\},\left\{K_{I_{e}}\right\}}^{c}(-1)^{\sum_{I_{a}}^{N_{a}} M_{I_{a}} \delta_{k_{I_{a}}}, 2+\sum_{I_{b}}^{N_{b}} M_{I_{b}} \delta_{k_{I_{b}}, 2}}\left(\prod_{I_{a}}^{N_{a}} z_{k_{I_{a}}, p_{I_{a}}}^{M_{I_{a}}} \prod_{I_{b}}^{N_{b}} w_{k_{I_{b}}, p_{I_{b}}}^{M_{I_{b}}} \prod_{I_{e}}^{N_{e}} \rho_{k_{I_{e}}, p_{I_{e}}}^{\left|M_{I_{e}}\right|+2 K_{I_{e}}}\right) \cos \left(\sum_{I_{e}}^{N_{e}} M_{I_{e}} \phi_{k_{I_{e}}}\right)
\end{aligned}
$$

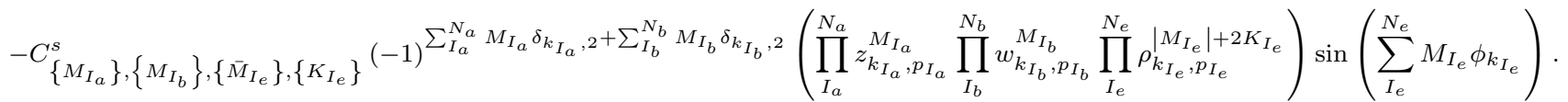

The $(-1)^{\sum_{I_{a}}^{N_{a}} M_{I_{a}} \delta_{k_{I}}, 2+\sum_{I_{b}}^{N_{b}} M_{I_{b}} \delta_{k_{I_{b}}}, 2}$ factor contains all possible sign flipping for the $a$ - and $b$-type coordinates, if their subscripts are 2. The minus sign of $-C_{\left\{M_{I_{a}}\right\},\left\{M_{I_{b}}\right\},\left\{\bar{M}_{I_{e}}\right\},\left\{K_{I_{e}}\right\}}$ arises from flipping the signs of all $\left\{\phi_{k_{I_{e}}}\right\}$ angles. The real and imaginary parts of the expansion above solely come from the real and imaginary parts of the $C^{c}=C^{c, r}+i C^{c, i}$ and $C^{s}=C^{s, r}+i C^{s, i}$ coefficients. To have $\chi_{R e}^{C_{2}^{\prime}}=1$ for this expansion, we need $\sum_{I_{a}}^{N_{a}} M_{I_{a}} \delta_{k_{I_{a}}, 2}+$ $\sum_{I_{b}}^{N_{b}} M_{I_{b}} \delta_{k_{I_{b}}, 2}=$ even in $C_{\left\{M_{I_{a}}\right\},\left\{M_{I_{b}}\right\},\left\{\bar{M}_{I_{e}}\right\},\left\{K_{I_{e}}\right\}}^{c, r}$ and odd in $C_{\left\{M_{I_{a}}\right\},\left\{M_{I_{b}}\right\},\left\{\bar{M}_{I_{e}}\right\},\left\{K_{I_{e}}\right\}}^{s, r}$. The opposite even/odd constraints give $\chi_{R e}^{C_{2}^{\prime}}=-1$. Transplanting the constraints onto $C_{\left\{M_{I_{a}}\right\},\left\{M_{I_{b}}\right\},\left\{\bar{M}_{I_{e}}\right\},\left\{K_{I_{e}}\right\}}^{c, i}$ and $C_{\left\{M_{I_{a}}\right\},\left\{M_{I_{b}}\right\},\left\{\bar{M}_{I_{e}}\right\},\left\{K_{I_{e}}\right\}}$ give the corresponding $\chi_{I m}^{C_{2}^{\prime}}$. These constraints and their resultant $\chi_{R e}^{C_{2}^{\prime}}$ and $\chi_{I m}^{C_{2}^{\prime}}$ are summarized in the "1 or -1 " row of Table 2. As shown in Table 1, when $\chi^{C_{n}}=1$ or -1 , the possible $\left(\chi_{R e}^{C_{2}^{\prime}}, \chi_{I m}^{C_{2}^{\prime}}\right)$ values are $(1,0),(-1,0),(1,-1)$, and $(-1,1)$. It is convenient to use Eq. 22 and Table 2 to obtain the expansions that fit the four sets of values. For the real-valued expansions with $\chi_{I m}^{C_{2}^{\prime}}=0$, we may just set all $\left\{C^{c, i}, C^{s, i}\right\}$ values to be zero.

Eq. 9 is used for expansions with $\chi^{C_{n}} \neq \pm 1$. Writing its real and imaginary parts of the expansion explicitly, Eq. 9 becomes

$$
\left(\prod_{I_{a}}^{N_{a}} z_{k_{I_{a}}, p_{I_{a}}}^{M_{I_{a}}} \prod_{I_{b}}^{N_{b}} w_{k_{I_{b}}, P_{I_{b}}}^{M_{I_{b}}} \prod_{I_{e}}^{N_{e}} \rho_{k_{I_{e}}, P_{I_{e}}}^{\left|M_{I_{e}}\right|+2 K_{I_{e}}}\right)\left[C_{\{\ldots\}}^{r} \cos \Phi-C_{\{\ldots\}}^{i} \sin \Phi+i\left(C_{\{\ldots\}}^{r} \sin \Phi-C_{\{\ldots\}}^{i} \cos \Phi\right)\right] .
$$


Table 2: Constraints of the $\chi^{C_{n} / S_{n}}$-eigenexpansions to have appropriate $\left(\chi_{R e}^{C_{2}^{\prime} / \sigma_{v}}, \chi_{I m}^{C_{2}^{\prime} / \sigma_{v}}\right)$ and $\chi^{\sigma_{h} / I}$.

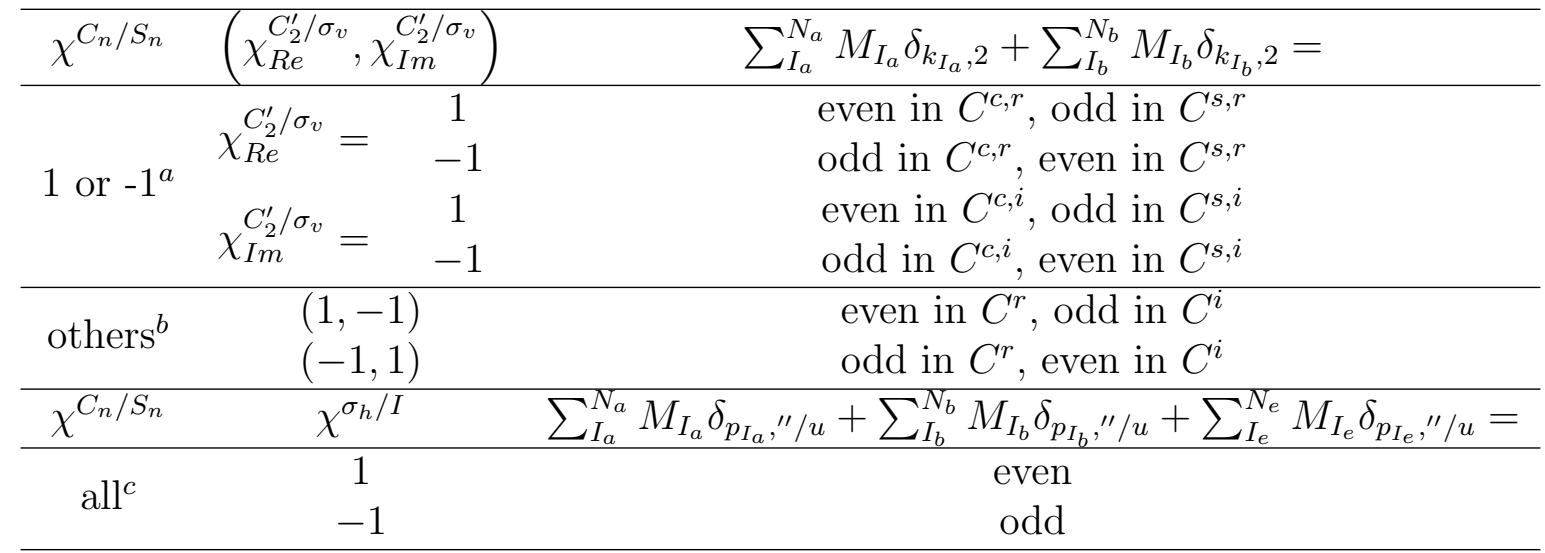

${ }^{a}$ The constraints apply to the expansion in Eq. 22. ${ }^{b}$ The constraints apply to the expansion in Eq. 9. ${ }^{c}$ The constraints apply to both Eqs. 9 and 22.

To save space, the subscripted indices sets of the coefficients are abbreviated as $\{\cdots\}$ and the total phase angle is abbreviated as $\Phi$, regardless of the values of the indices. Under $\hat{C}_{2}^{\prime}$, the expansion becomes

$$
\begin{array}{r}
(-1)^{\sum_{I_{a}}^{N_{a}} M_{I_{a}} \delta_{k_{I_{a}}, 2}+\sum_{I_{b}}^{N_{b}} M_{I_{b}} \delta_{k_{I_{b}}, 2}}\left(\prod_{I_{a}}^{N_{a}} z_{k_{I_{a}}, p_{I_{a}}}^{M_{I_{a}}} \prod_{I_{b}}^{N_{b}} w_{k_{I_{b}}, p_{I_{b}}}^{M_{I_{b}}} \prod_{I_{e}}^{N_{e}} \rho_{k_{I_{e}}, p_{I_{e}}}^{\left|M_{I_{e}}\right|+2 K_{I_{e}}}\right) \\
{\left[C_{\{\cdots\}}^{r} \cos \Phi+C_{\{\cdots\}}^{i} \sin \Phi+i\left(-C_{\{\cdots\}}^{r} \sin \Phi-C_{\{\cdots\}}^{i} \cos \Phi\right)\right] .}
\end{array}
$$

Again, the $(-1)^{\sum_{I_{a}}^{N_{a}} M_{I_{a}} \delta_{k_{I}, 2}+\sum_{I_{b}}^{N_{b}} M_{I_{b}} \delta_{k_{I_{b}}}, 2}$ factor comes from possible sign changes for the $a$ - and $b$-type coordinates, and the sign changes in front of the sin $\Phi$ terms come from flipping the signs of all $\left\{\phi_{k_{I}}\right\}$ angles. As shown in Table 1 , the only possible $\left(\chi_{R e}^{C_{2}^{\prime}}, \chi_{I m}^{C_{I}^{\prime}}\right)$ combinations for the matrix elements with $\chi^{C_{n}} \neq \pm 1$ are $(1,-1)$ and $(-1,+1)$. For the former, $\sum_{I_{a}}^{N_{a}} M_{I_{a}} \delta_{k_{I_{a}}, 2}+\sum_{I_{b}}^{N_{b}} M_{I_{b}} \delta_{k_{I_{b}}, 2}$ needs to be even in $C_{\left\{M_{I_{a}}\right\},\left\{M_{I_{b}}\right\},\left\{M_{I_{e}}\right\},\left\{K_{I_{e}}\right\}}^{r}$ and odd in $C_{\left\{M_{I_{a}}\right\},\left\{M_{I_{b}}\right\},\left\{M_{I_{e}}\right\},\left\{K_{I_{e}}\right\}}$. For the latter, the opposite evenness-oddness is needed. These constraints are summarized in the "others" row of Table 2. 
Each term in Eqs. 9 and 22 is a $\hat{\sigma}_{h}$-eigenfunction with $\chi^{\sigma_{h}}=(-1)^{\sum_{I_{a}}^{N_{a}} M_{I_{a}} \delta_{p_{I}},{ }^{\prime \prime}+\sum_{I_{b}}^{N_{b}} M_{I_{b}} \delta_{P_{I_{b}}},{ }^{\prime \prime}+\sum_{I_{e}}^{N_{e}} M_{I_{e}} \delta_{p_{I_{e}}}, "}$. Therefore, to have an expansion with $\chi^{\sigma_{h}}=1$ or -1 , only those terms with $\sum_{I_{a}}^{N_{a}} M_{I_{a}} \delta_{p_{I_{a}}}, "+\sum_{I_{b}}^{N_{b}} M_{I_{b}} \delta_{p_{I_{b}}},{ }^{\prime \prime}+\sum_{I_{e}}^{N_{e}} M_{I_{e}} \delta_{p_{I_{e}},},=$ even or odd, respectively, shall be kept. These constraints are included in the "all" row of Table 2. It is never tedious to emphasize that the constraints for obtaining expansions with $\chi^{C_{n}}$ above are also applicable for obtaining expansions with $\chi^{S_{n}}$ in $S_{n_{e}}$ and $D_{2 n_{e} d}$ symmetries. In those symmetries, the principal axes are $S_{n_{e}}$ and $S_{2 n_{e}}$, respectively. The constraints for obtaining expansions with $\left(\chi_{R e}^{C_{2}^{\prime}}, \chi_{I m}^{C_{2}^{\prime}}\right)$ are applicable for obtaining expansions with $\left(\chi_{R e}^{\sigma_{v}}, \chi_{I m}^{\sigma_{v}}\right)$ in $C_{n v}$ symmetries. The constraints for obtaining expansions with $\chi^{\sigma_{h}}$ are applicable for obtaining expansions with $\chi^{I}$ in $C_{n_{e} h}, D_{n_{e} h}$, and $D_{n_{o} d}$ symmetries. In Tables 1 and 2, the slash "/" is used to indicate the multiple symmetry elements to which the symmetry-eigenvalues and constraints are applicable.

The three equations $(9,22$, and 21$)$ and the two tables conveys a unified formalism for all $\mathrm{JT} / \mathrm{pJT}$ vibronic Hamiltonians in axial symmetries. It is more concise than those separately derived before for trigonal and tetragonal symmetries. The trigonal formalism ${ }^{44}$ consists of 1 table of symmetry-eigenvalues, 2 tables of expansion formulas, and 4 tables of constraints. The corresponding numbers are 1, 3, and 6 in the tetragonal formalism. ${ }^{45}$ Also, the previous formalisms are for bimodal problems only, while the present one is for an arbitrary number of modes of arbitrary types.

\subsection{Renner-Teller problems}

Like JT/pJT interactions in systems with axial symmetries, vibronic interactions in linear molecules involving degenerate vibrational modes result in orbital degeneracy lifting. The associated effects are called the Renner-Teller (RT) effects. ${ }^{57,58}$ The $C_{\infty v}$ or $D_{\infty h}$ symmetries of linear molecules are special cases of axial symmetries. Therefore, the presented formalism must cover Renner-Teller problems, with the limit of $n \rightarrow \infty$. In a linear molecule, each atom can only move along or perpendicular the molecular axis. Therefore, all vibrational 
modes must transform as $\Sigma^{+}$or $\Pi$ IRREP, corresponding to $a_{1}$-type and $e_{1}$-type modes in the present formalism. Eqs. 9 and 22 are thus simplified to

$$
\begin{array}{r}
C_{\left\{M_{I_{a}}\right\},\left\{M_{I_{e}}\right\},\left\{K_{I_{e}}\right\}}\left(\prod_{I_{a}}^{N_{a}} z_{1_{I_{a}}, p_{I_{a}}}^{M_{I_{a}}} \prod_{I_{e}}^{N_{e}} \rho_{1_{I_{e}}, p_{I_{e}}}^{\left|M_{I_{e}}\right|+2 K_{I_{e}}}\right) e^{i\left(\sum_{I_{e}}^{N_{e}} M_{I_{e}} \phi_{1_{I_{e}}}\right)} \\
C_{\left\{M_{I_{a}}\right\},\left\{\bar{M}_{I_{e}}\right\},\left\{K_{I_{e}}\right\}}^{c}\left(\prod_{I_{a}}^{N_{a}} z_{1_{I_{a}}, p_{I_{a}}}^{M_{I_{a}}} \prod_{I_{e}}^{N_{e}} \rho_{1_{I_{e}}, p_{I_{e}}}^{\left|M_{I_{e}}\right|+2 K_{I_{e}}}\right) \cos \left(\sum_{I_{e}}^{N_{e}} M_{I_{e}} \phi_{1_{I_{e}}}\right) \\
+C_{\left\{M_{I_{a}}\right\},\left\{\bar{M}_{I_{e}}\right\},\left\{K_{I_{e}}\right\}}^{s}\left(\prod_{I_{a}}^{N_{a}} z_{1_{I_{a}}, p_{I_{a}}}^{M_{I_{a}}} \prod_{I_{e}}^{N_{e}} \rho_{1_{I_{e}}, p_{I_{e}}}^{\left|M_{I_{e}}\right|+2 K_{I_{e}}}\right) \sin \left(\sum_{I_{e}}^{N_{e}} M_{I_{e}} \phi_{1_{I_{e}}}\right) .
\end{array}
$$

Eq. 21 becomes

$$
\sum_{I_{e}}^{N_{e}} M_{I_{e}}=-\kappa .
$$

The $L n$ in Eq. 21 is dropped since the summation of the finite $\left\{M_{I_{e}}\right\}$ values never reaches the infinite $L n$. The $\Sigma^{+}$and $\Sigma^{-}$-type electronic states correspond to $A_{1}$ - and $A_{2}$-type states, and the other states characterized by $\Lambda$ correspond to the $E_{k}$-type states with $k=\Lambda$, e.g., П, $\Delta$ corresponding to $E_{1}, E_{2}$, etc. Since $n \rightarrow \infty, B$-type states with $\Lambda=\frac{n}{2}$ can never be reached. With all these simplifications and correspondences, the unified formalism can be used to obtain Hamiltonian expansion formulas for all RT problems.

\subsection{Rules for linear coupling}

Linear vibronic coupling in JT interactions is of special interest. It makes a high-symmetry configuration a conical intersection on adiabatic potential energy surface and and leads to geometric phase effects. ${ }^{59-61}$ For a degenerate $E_{k}$-type term symbol, $\chi^{C_{n}}=1, e^{i \frac{4 k \pi}{n}}(\kappa=0,2 k)$ in Table 1 for the two unique matrix elements. According to Eq. 21, the linear coupling mode must be an $e_{l}$-type mode that has $\pm l=L n$ or $L n-2 k$, or a $b$-type mode when $\frac{n_{e}}{2}=L n_{e}$ or $L n_{e}-2 k \cdot \frac{n_{e}}{2}=L n_{e}$ is certainly impossible except for the non-existing case of $n_{e}=0$. Also, $\pm l=L n$ is impossible given the range of $l=1,2, \cdots \operatorname{Int}\left(\frac{n_{o}}{2}\right)$ or $\frac{n_{e}}{2}-1$. The only 
possibility for $\frac{n_{e}}{2}=L n_{e}-2 k$ is $k=\frac{n_{e}}{4}$. This is why the $E$-type terms of tetragonal systems feature linear JT interactions along $b$-type modes, hexagonal systems do not have linear JT interactions along $b$-modes, and octagonal systems only have their $E_{2}$-type terms have linear couplings along $b$-type modes. Only $4 n$-gonal systems have their $E_{n}$ terms feature linear JT interactions along $b$-type modes. The range of $l$ reduces $\pm l=L n-2 k$ to $l=n-2 k$ or $2 k$. This explains the $E \otimes e$ linear JT interaction in trigonal systems $(l=1, k=1, n=3)$, the lack of this type in tetragonal systems $(l=1, k=1, n=3)$, and the $E_{2} \otimes e_{1}$-type linear JT interactions in pentagonal systems.

The $\chi^{\sigma_{h} / I}=1$ for $H_{+_{k, p}-k, p}$ determines that in axial symmetries where there are $\sigma_{h}$ or $I$, only the modes have even $\hat{\sigma}_{h} / \hat{I}$-parity can give linear JT interactions. In all cases of $\left(\chi_{R e}^{C 2^{\prime} / \sigma_{v}}, \chi_{I m}^{C 2^{\prime} / \sigma_{v}}\right)$ in Table 2, the odd constraint applies to at least one set of coefficients. Therefore, both $b_{1}$ - and $b_{2}$-type modes can give linear JT interactions in axial symmetries with $\sigma_{v}$ or $C_{2}^{\prime}$. There is also no restriction onto $e_{l}$-type modes due to the presence of $\sigma_{v}$ or $C_{2}^{\prime}$. All rules for the existence of linear JT interactions in axial symmetries are included in these two paragraphs.

As to linear molecules, only $e_{l=1}$-type modes are available for distorting (bending) structure. The $\pm l=L n-2 k$ condition is reduced to $2 k=1$, which is impossible. There is hence no linear vibronic coupling within a degenerate term symbol in linear molecules, and a linear structure that undergoes bending has a maximum (instead of a conical intersection) of its ground state potential energy surface when it is unbent. The $\chi^{C_{n}}$ values in Table 1 indicate that linear vibronic coupling in linear molecules can only occur between states with their $\Lambda$ values differing by \pm 1 , i.e., a type of pJT interaction, which is essentially the only driving force for bending a linear molecule, as the RT bending propensity is generally weak. ${ }^{62}$ Many of the aforementioned rules are well known. The present formalism elucidates them in a very clear way and more importantly, provides quick and correct predictions for the (non-)existence of vibronic couplings at certain orders. 


\subsection{An Example}

We use a $E_{3}^{\prime \prime} \otimes e_{1}^{\prime}$ problem of a heptagonal system to demonstrate the use and correctness of the presented formalism. The system is chosen to be the cycloheptatrienyl cation $\left(\mathrm{C}_{7} \mathrm{H}_{7}^{+}\right)$. Its degenerate $\mathrm{HOMO}+3,4$ are chosen as the two components of a single-electron $E_{3}^{\prime \prime}$ state. The $e_{1}^{\prime}$ mode of $1012 \mathrm{~cm}^{-1}$ is selected. The $\mathrm{C}_{7} \mathrm{H}_{7}^{+}$structure, orbitals, and vibrational modes were obtained at the B3LYP/cc-pVDZ level of calculation. The orbitals and component modes are shown in Figure 1(a). The orbitals are frozen so that they are strict diabatic states. We choose such a heptagonal system, instead of those with lower $n$ values, to emphasize that the formalism is applicable to arbitrarily high axial symmetries.

For such a unimodal problem, Eq. 9 is reduced to

$$
C_{M, K} \rho_{1}^{|M|+2 K} e^{i M \phi_{1}}
$$

which is applicable to both $H_{+_{3}^{\prime \prime}+_{3}^{\prime \prime}}$ and $H_{+_{3}^{\prime \prime}-{ }_{3}^{\prime \prime}}$, the only two matrix elements according to the $E_{k, p}$ row in Table 1 . We focus on the $H_{+_{3}^{\prime \prime}-_{3}^{\prime \prime}}$ element. The $E_{k, p}$ row in Table 1 indicates that this matrix element features $\chi^{C_{n}},\left(\chi_{R e}^{C_{2}^{\prime}}, \chi_{I m}^{C_{2}^{\prime}}\right)=e^{i \frac{12 \pi}{7}},(1,-1)$. With $\kappa=6$, $N_{b}=0, N_{e}=1, k_{1}=1$, and $n=7$, Eq. 21 becomes $M=7 L-6$. Certainly, only $M=1,-6,8,-13,15, \cdots$ are kept in Eq. 29 in the expansion for $H_{+{ }_{3}^{\prime \prime}-_{3}^{\prime \prime}}$. Since $N_{a}=N_{b}=0$,

$\sum_{I_{a}}^{N_{a}} M_{I_{a}} \delta_{k_{I_{a}}, 2}+\sum_{I_{b}}^{N_{b}} M_{I_{b}} \delta_{k_{I_{b}}, 2}=0$. Given this even number and $\chi^{C_{n}} \neq \pm 1$, Table 2 indicates that $C_{M, K}$ in Eq. 29 must be real-valued to expand $H_{++_{3}^{\prime \prime}-_{3}^{\prime \prime}}$ :

$$
H_{+_{3}^{\prime \prime}-_{3}^{\prime \prime}}=C_{M, K}^{r} \rho_{1}^{|M|+2 K} e^{i M \phi_{1}}
$$

In the more commonly used real-valued electronic bases $\left|X_{3}^{\prime \prime}\right\rangle$ and $\left|Y_{3}^{\prime \prime}\right\rangle$ (see Eq. 2 for the transformation), the real and imaginary parts of $H_{+_{3}^{\prime \prime}-{ }_{3}^{\prime \prime}}$ become

$$
\frac{H_{X_{3}^{\prime \prime} X_{3}^{\prime \prime}}-H_{Y_{3}^{\prime \prime} Y_{3}^{\prime \prime}}}{2}=C_{M, K}^{r} \rho_{1}^{|M|+2 K} \cos \left(M \phi_{1}\right) ; H_{X_{3}^{\prime \prime} Y_{3}^{\prime \prime}}=-C_{M, K}^{r} \rho_{1}^{|M|+2 K} \sin \left(M \phi_{1}\right)
$$




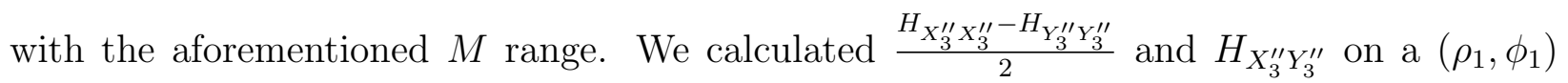
grid, and then expand them into Fourier series of $\sin \left(M \phi_{1}\right)$ and $\cos \left(M \phi_{1}\right)$ at each of the $\rho_{1}$ grid point. The expansion coefficients were obtained by numerically integrating $\phi_{1}$ from

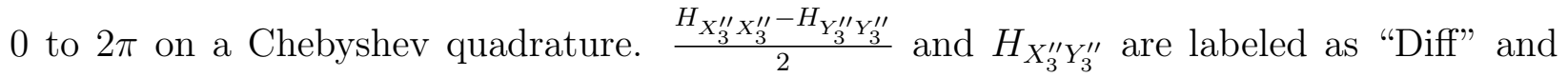
"Off" terms henceforth, for they are the difference between diagonal and the off-diagonal element, respectively. The Diff and Off expansion coefficients discussed below are obtained from the calculated Diff and Off values independently, without assuming any magnitude and sign relations between the two sets. The resultant sign and magnitude relations confirm the relations predicted by the formalism.

(a)

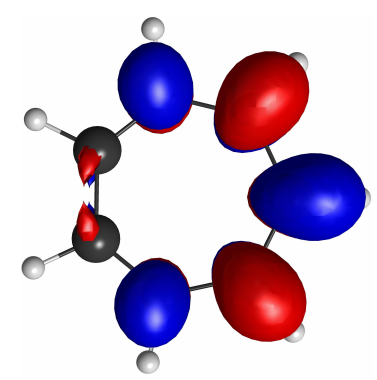

$E^{\prime \prime} 3, x$

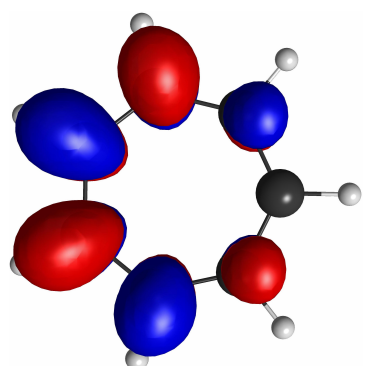

$E^{\prime \prime} 3, y$

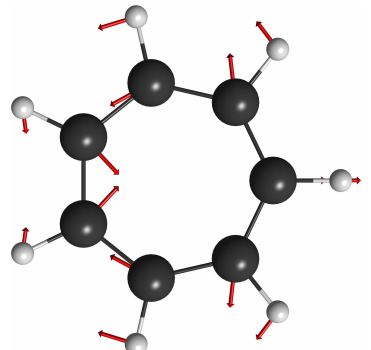

$e^{\prime}{ }_{1, x}$

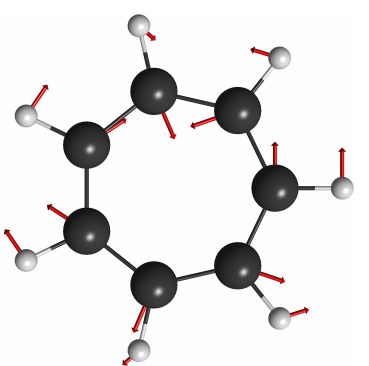

$e^{\prime}{ }_{1, y}$

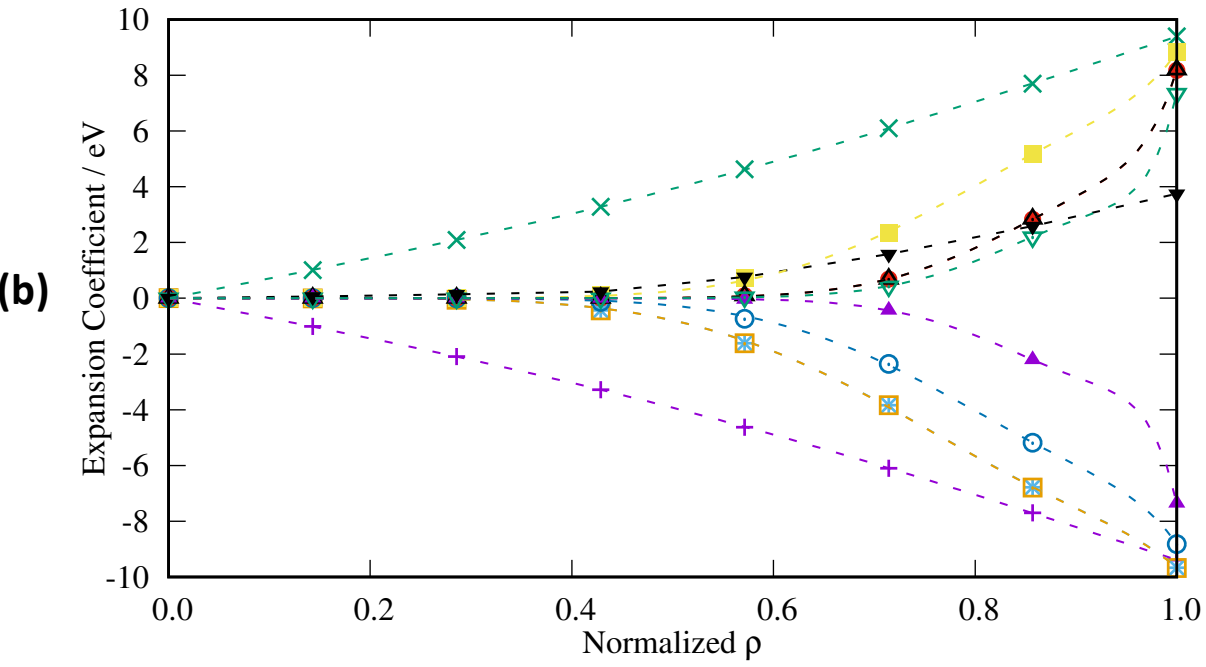

Diff $\cos \left(\phi_{1}\right)+$ Off $\sin \left(\phi_{1}\right) \times$ Diff $\cos \left(6 \phi_{1}\right) \times 40 \quad \%$ Off $\sin \left(6 \phi_{1}\right) \times 40$ Diff $\cos \left(8 \phi_{1}\right) \times 140$ Off $\sin \left(8 \phi_{1}\right) \times 140 \circ$ Diff $\cos \left(13 \phi_{1}\right) \times 600$ Off $\sin \left(13 \phi_{1}\right) \times 600 \Delta$ Diff $\cos \left(15 \phi_{1}\right) \times 1500$ ॥ Off $\sin \left(15 \phi_{1}\right) \times 1500 \nabla$ Diff others x 1500 v

Figure 1: (a) The $\mathrm{HOMO}+3,4$ orbitals and the $e_{1}^{\prime}$ component modes of $\mathrm{C}_{7} \mathrm{H}_{7}^{+}$used to demonstrate the formalism; (b) The Fourier series coefficients for $\frac{H_{X_{3}^{\prime \prime} X_{3}^{\prime \prime}}-H_{Y_{3}^{\prime \prime} Y_{3}^{\prime \prime}}}{2}$ (labeled as "Diff") and $H_{X_{3}^{\prime \prime} Y_{3}^{\prime \prime}}$ (labeled as "Off'). The coefficients are scaled so that they can be compared in the same graph. 
$\sin \left(M \phi_{1}\right)$ and $\cos \left(M \phi_{1}\right)$ terms up to $M=18$ are included in the Diff and Off expansions at each $\rho$. Shown by the markers in Figure 1(b) are the expansion coefficients that are substantial in the range of distortion $\left(\rho_{\max }=6.3 \sqrt{\mathrm{amu}} \AA\right)$. By substantial, we also mean the rate of magnitude increase as $\rho$ becomes larger. For instance, the coefficients for the $M=13,15$ terms are small throughout the $\rho$ range and need to be amplified by 600 and 1500 times to be comparable with the $M=1$ coefficients. However, their magnitudes increase sharply as $\rho$ increases. The unsubscripted $\rho$ coordinate in the figure is renormalized as $\rho_{1} / \rho_{\max }$ and has no unit. The Diff and Off expansions only take $\cos \left(M \phi_{1}\right)$ terms and $\sin \left(M \phi_{1}\right)$ terms, respectively, with $M=1,6,8,13,15$. The magnitude of coefficients of all the other $\cos \left(M \phi_{1}\right)$ and $\sin \left(M \phi_{1}\right)$ terms, which is calculated as the square root of the summation of their squares, is plotted in Figure 1(b) and labeled as "others". The Diff "others" and almost identical to the Off analogues and only the formers are plotted. The "others" coefficients remain small throughout the range of distortion and never rise up quickly as the $M=13,15$ coefficients. The magnitudes of the corresponding cos and sin coefficients for Diff and Off are essentially identical. They have the opposite signs for $M=1,8,15$, which is consistent with the minus sign for the Off expansion in Eq. 31. They have the same sign for $M=6,13$, and this is because it is $M=-6,-13$ that enter Eq. 31; the minus sign does not affect the cos terms in the Diff expansion, and it is cancelled by the minus sign for the Off expansion. The correctness of the angular factors in Eq. 31 and the $M=1,-6,8,-13,15, \cdots$ selection are fully confirmed. Note that for the $E \otimes e$ problem in the lower trigonal symmetry, the selection of $M$ for $H_{+-}$is $M=-1,2,-4,5,-7,8, \cdots$. As expected, high symmetry imposes more constrains on Hamiltonian expansion, following the same logics as the constraints in Table 2. For the larger $n$, the constraint is hidden in the larger periodicity of $L n$ in Eq. 21.

The dashed curves shown in Figure 1(b) are the fitted curves for each set of the Fourier coefficients. We use a three terms polynomial $a_{M} \rho^{M}+a_{M+2} \rho^{M+2}+a_{M+4} \rho^{M+4}$ to fit the $\cos \left(M \phi_{1}\right)$ coefficients with one $M$ (always taken to be positive as in the figure). Another 
fitting was done for the corresponding $\sin \left(M \phi_{1}\right)$ coefficients. For instance, the resultant polynomials for $\cos \left(\phi_{1}\right)$ and $\sin \left(\phi_{1}\right)$ coefficients are

$$
-7.05969 \rho-3.46937 \rho^{3}+1.14006 \rho^{5} ; 7.06001 \rho+3.47178 \rho^{3}-1.13934 \rho^{5}
$$

respectively. As expected, the magnitude and sign relations between the Diff and Off expansion coefficients are maintained in the two polynomials above, and in all the other pairs (given in Section S.3). The three terms fittings give very small errors in this large range of distortion, as shown by the consistence between the curves and the markers in Figure 1(b).

This consistence confirms the adequacy of the $\rho_{1}^{|M|+2 K}$ power selection with just lowest order terms. We do not need to prove the correctness of this radial coordinate power selection using a free fitting, as for the angular factors. This is because the power selection is ensured by the smooth variation of the calculated data around $\rho=0$, i.e., the fitted function must be a differentiable functions of the associated Cartesian coordinates.

\section{VHEGEN2.0}

A Python program called VHEGEN (Vibronic Hamiltonian Expansion GENerator) was developed to generate expansion formulas for all bimodal and unimodal pJT/JT Hamiltonians in trigonal and tetragonal symmetries. ${ }^{63}$ The 2.0 version of the program has been extended to allow any axial symmetries and any numbers and types of modes. Instead of having specific root formulas and constrain tables for each state combination, the general root formulas of Eqs. 9, 22, 26 and 27 are used in the program, along with Tables 1 and 2. The package can be installed through pip install vhegen. After installation, the program can be called as described in Ref. 63 or by using the interactive script vhegen in command line.

In order to efficiently obtain the Hamiltonian expansions for a given order $o$, a constrained summation ${ }^{64}$ approach has been used to select the appropriate mode combinations. This attenuates the exponential scaling with the number of modes $m$ and order $o$ such that the 
search space reduces from $o^{m}$ to $\frac{(o+m) !}{m ! o !}$. With this problem being solved, the main bottleneck of the program is the symbolic conversion from polar to Cartesian functions. This conversion is performed by the sympy package with the substitution $\phi \rightarrow \operatorname{atan} 2(y, x), \rho \rightarrow \sqrt{x^{2}+y^{2}}$ for each e-mode followed by an expand command.

The program has also been extended to output two $\mathrm{MCTDH}^{65}$ operator files such that one can simulate the dynamics of the system in either polar or Cartesian coordinates. The Laplace operator for polar coordinates is described in Section S.4. The summation of $\phi$ coordinates in the polar expansion's sin and cos terms have been further expanded such that the generated operator is in the necessary sum-of-products form. For each set of polar e-mode coordinates, the generalized Laguerre polynomials with $\alpha=1$ (lagu1 in MCTDH) shall be used as radial basis and either exponential or FFT functions with a range of $[0,2 \pi)$ as polar angle basis. For Cartesian coordinates, harmonic oscillator basis functions are a good choice. VHEGEN2.0 outputs an MCTDH input file with reasonable basis function parameters made.

As an example, the program is used to generate the $E_{1 g} \otimes\left(2 e_{2 g}+e_{1 g}+e_{1 u}+e_{2 u}\right)$ Hamiltonian in $D_{6 h}$ from zero to sixth order. This is a two-state, ten-mode problem that one would see in the benzene cation. The program generates the Hamiltonian and outputs the pdf and MCTDH input files in about 15 minutes on a desktop workstation. The generated expansion satisfies all the constraints listed above and includes 1092 parameters. This compares to 32032 parameters in the full series expansion (including terms that are not of the correct symmetry) with order $\leq 6$. A step-by-step instruction of obtaining the program and running it for the example is given in Section S.5.

\section{CONCLUSIONS}

There have been numerous endeavours to derive high order expansion formulas for JT/pJT Hamiltonians. Usually, these attempts are performed in a case by case, and order by order 
manner. In this work, we derive and present a unified Hamiltonian formalism for JT/pJT problems in all axial symmetries. The formalism is inclusive, as it covers all axial JT/pJT problems, with all possible combinations of electronic states, with an arbitrary number of all types of vibrational modes, covering all axial symmetries from $C_{1}$ to $D_{\infty h}$. Of likewise importance, it gives Hamiltonian expansions up to arbitrary orders. The formalism is concise and easy to use. It essentially consists of three equations and two tables. The correctness and convenience of the formalism is demonstrated using a $E_{3}^{\prime \prime} \otimes e_{1}^{\prime}$ problem in a heptagonal system. The formalism is programmable, as it involves looking up the tables and imposing constraints on the generic expansion formulas. A Python code, VHEGEN2.0, is developed to generate any desired Hamiltonian expansion formulas based on the simplest input. The program also interfaces the expansion formulas to the quantum dynamics simulation program MCTDH. This work has significantly simplified future studies of pJT/JT problems in high order axial symmetries. The knowledge gained in this study is of critical importance for future works in deriving JT/pJT Hamiltonians for cubic group systems, and spin-orbit JT/pJT Hamiltonians for axial and cubic group systems.

\section{ACKNOWLEDGEMENTS}

T.Z. is thankful to the Natural Sciences and Engineering Research Council (NSERC) of Canada for research funding (RGPIN-2016-06276) and Carleton University for start-up grant (186853). 


\section{References}

(1) Englman, R. Jahn-Teller Effect in Molecules and Crystals; John Wiley and Sons, Ltd.: London, 1972.

(2) Jahn, H. A.; Teller, E. Stability of Polyatomic Molecules in Degenerate Electronic States. Proc. Roy. Soc. A 1937, 161, 220-235.

(3) Bersuker, I. B.; Polinger, V. Z. Vibronic Interactions in Molecules and Crystals; Springer-Verlag, 1989.

(4) Bersuker, I. B. The Jahn-Teller Effect; Cambridge University Press: Cambridge, UK, 2006.

(5) Öpik, U.; Pryce, M. H. L. Studies of the Jahn-Teller Effect. I. A Survey of the Static Problem. Proc. R. Soc. A 1957, 238, 425-447.

(6) Bersuker, I. B. Spontaneous Symmetry Breaking in Matter Induced by Degeneracies and Pseudodegeneracies. Adv. Chem. Phys. 2016, 160, 159-208.

(7) Barckholtz, T. A.; Miller, T. A. Quantitative Insights about Molecules Exhibiting JahnTeller and Related Effects. Int. Rev. Phys. Chem. 1998, 17, 435-524.

(8) Goodenough, J. B. Jahn-Teller Phenomena in Solids. Ann. Rev. Mater. Sci. 1998, 28, $1-27$.

(9) Bersuker, I. B. Modern Aspects of the Jahn-Teller Effect Theory and Applications To Molecular Problems. Chem. Rev. 2001, 101, 1067-1114.

(10) Applegate, B. E.; Barckholtz, T. A.; Miller, T. A. Explorations of Conical Intersections and Their Ramifications for Chemistry through the Jahn-Teller Effect. Chem. Soc. Rev. 2003, 32, 38-49. 
(11) Köppel, H. In Conical intersections: electronic structure, dynamics and spectroscopy; Domcke, W., Yarkony, D. R., Köppel, H., Eds.; World Scientific: New Jersey, 2004; Chapter 10, pp 429-472.

(12) Halcrow, M. A. Jahn-Teller Distortion in Transition Metal Compounds, and Their Importance in Functional Molecular and Inorganic Materials. Chem. Soc. Rev. 2013, 42, 1784-1795.

(13) Bersuker, I. B. Pseudo-Jahn-Teller Effect-A Two-State Paradigm in Formation, Deformation, and Transformation of Molecular Systems and Solids. Chem. Rev. 2013, 113, $1351-1390$.

(14) Hermoso, W.; Liu, Y.; Bersuker, I. B. Novel Effect Induced by Pseudo-Jahn-Teller Interactions: Broken Cylindrical Symmetry in Linear Molecules. J. Chem. Theory Comput. 2014, 10, 4377-4388.

(15) Clemente-Juan, J. M.; Palii, A.; Coronado, E.; Tsukerblat, B. Mixed-Valence Molecular Unit for Quantum Cellular Automata: Beyond the Born-Oppenheimer Paradigm through the Symmetry-Assisted Vibronic Approach. J. Chem. Theory Comput. 2016, 12, 3545-3560.

(16) Kim, I.; Jeon, S. O.; Jeong, D.; Choi, H.; Son, W.-J.; Kim, D.; Rhee, Y. M.; Lee, H. S. Spin-Vibronic Model for Quantitative Prediction of Reverse Intersystem Crossing Rate in Thermally Activated Delayed Fluorescence Systems. J. Chem. Theory Comput. 2020, 16, 621-632.

(17) Toutounji, M. Spectroscopy of Vibronically Coupled and Duschinskcally Rotated Polyatomic Molecules. J. Chem. Theory Comput. 2020, 16, 1690-1698.

(18) Bersuker, I. B. Jahn-Teller and Pseudo-Jahn-Teller Effects: From Particular Features to General Tools in Exploring Molecular and Solid State Properties. Chem. Rev. 2021, 121, 1463-1512. 
(19) Bersuker, I. B.; Stavrov, S. S. Structure and Properties of Metalloporphyrins and Hemoproteins: the Vibronic Approach. Coord. Chem. Rev. 1988, 88, 1-68.

(20) Köppel, H. In Conical intersections: electronic structure, dynamics and spectroscopy; Domcke, W., Yarkony, D. R., Köppel, H., Eds.; World Scientific: New Jersey, 2004; Chapter 4, pp 175-204.

(21) Domcke, W.; Woywod, C. Direct Construction of Diabatic States in the CASSCF Approach. Application to the Conical Intersection of the ${ }^{1} A_{2}$ and ${ }^{1} B_{1}$ Excited States of Ozone. Chem. Phys. Lett. 1993, 216, 362-368.

(22) Ichino, T.; Gauss, J.; Stanton, J. F. Quasidiabatic states described by coupled-cluster theory. J. Chem. Phys. 2009, 130, 174105.

(23) Köppel, H.; Gronki, J.; Mahapatra, S. Construction Scheme for Regularized Diabatic States. J. Chem. Phys. 2001, 115, 2377-2388.

(24) Naskar, K.; Mukherjee, S.; Mukherjee, B.; Ravi, S.; Mukherjee, S.; Sardar, S.; Adhikari, S. ADT: A Generalized Algorithm and Program for Beyond Born-Oppenheimer Equations of " $N$ " Dimensional Sub-Hilbert Space. J. Chem. Theory Comput. 2020, $16,1666-1680$.

(25) Yin, Z.; Braams, B. J.; Fu, B.; Zhang, D. H. Neural Network Representation of ThreeState Quasidiabatic Hamiltonians Based on the Transformation Properties from a Valence Bond Model: Three Singlet States of $\mathrm{H}_{3}^{+}$. J. Chem. Theory Comput. 2021, 17, 1678-1690.

(26) Viel, A.; Eisfeld, W. Effects of Higher Order Jahn-Teller Coupling on the Nuclear Dynamics. J. Chem. Phys. 2004, 120, 4603-4613.

(27) Eisfeld, W.; Viel, A. Higher Order $(A+E) \times e$ Pseudo-Jahn-Teller Coupling. J. Chem. Phys. 2005, 122, 204317. 
(28) Mahapatra, S.; Eisfeld, W.; Köppel, H. Effects of Multimode Jahn-Teller Coupling on the Photodetachment Spectrum of Nitrate Anion $\left(\mathrm{NO}_{3}^{-}\right)$. Chem. Phys. Lett. 2007, 441, $7-15$.

(29) Bhattacharyya, S.; Opalka, D.; Poluyanov, L. V.; Domcke, W. Jahn-Teller Theory beyond the Standard Model. J. Phys. Conf. Series 2013, 428, 012015.

(30) Bhattacharyya, S.; Opalka, D.; Poluyanov, L. V.; Domcke, W. The $(E+A) \times(e+a)$ Jahn-Teller and Pseudo-Jahn-Teller Hamiltonian Including Spin-Orbit Coupling for Trigonal Systems. J. Phys. Chem. A 2014, 118, 11962-11970.

(31) Eisfeld, W.; Vieuxmaire, O.; Viel, A. Full-Dimensional Diabatic Potential Energy Surfaces Including Dissociation: the ${ }^{2} E^{\prime \prime}$ State of $\mathrm{NO}_{3}$. J. Chem. Phys. 2014, 140, 224109.

(32) Codd, T.; Chen, M.-W.; Roudjane, M.; Stanton, J. F.; Miller, T. A. Jet Cooled Cavity Ringdown Spectroscopy of the $\tilde{A}^{2} E^{\prime \prime} \leftarrow \tilde{X}^{2} A_{2}^{\prime}$ Transition of the $\mathrm{NO}_{3}$ Radical. J. Chem. Phys. 2015, 142, 184305.

(33) Mondal, T. On the higher-order $T_{2} \otimes\left(e+t_{2}\right)$ Jahn-Teller coupling effects in the photodetachment spectrum of the alanate anion $\left(\mathrm{AlH}_{4}^{+}\right)$. Phys. Chem. Chem. Phys. 2018, 20, 9401-9410.

(34) Tran, H. K.; Stanton, J. F.; Miller, T. A. Quantifying the Effects of Higher Order Coupling Terms on Fts Using a Second Order Jahn-Teller Hamiltonian. J. Mol. Spectrosc. 2018, 343, 102-115.

(35) Weaver, A.; Arnold, D. W.; Bradforth, S. E.; Neumark, D. M. Examination of the ${ }^{2} A_{2}^{\prime}$ and ${ }^{2} E^{\prime}$ States of $\mathrm{NO}_{3}$ by Ultraviolet Photoelectron Spectroscopy of $\mathrm{NO}_{3}^{-}$. J. Chem. Phys. 1991, 94, 1740-1751.

(36) Shao, Z.; Li, H.; Zhang, S.; Li, J.; Dai, Z.; Mo, Y. The Jahn-Teller Effect in 
$\mathrm{CH}_{3} \mathrm{Cl}^{+}\left(\tilde{X}^{2} E\right)$ : A Combined High-Resolution Experimental Measurement and ab initio Theoretical Study. J. Chem. Phys. 2012, 136, 064308.

(37) Sharma, K.; Garner, S.; Miller, T. A.; Stanton, J. F. First-Principles Calculation of JahnTeller Rotational Distortion Parameters. J. Phys. Chem. A 2019, 123, 4990-5004.

(38) Sharma, K.; Miller, T. A.; Stanton, J. F. Vibronically Coupled States: Computational Considerations and Characterisation of Vibronic and Rovibronic Spectroscopic Parameters. Int. Rev. Phys. Chem. 2021, 40, 165-298.

(39) Opalka, D.; Domcke, W. High-Order Expansion of $T_{2} \times t_{2}$ Jahn-Teller Potential-Energy Surfaces in Tetrahedral Molecules. J. Chem. Phys. 2010, 132, 154108.

(40) Opalka, D.; Domcke, W. High-Order Expansion of $T_{2} \otimes e$ Jahn-Teller Potential-Energy Surfaces in Tetrahedral Systems. Chem. Phys. Lett. 2010, 494, 134-138.

(41) Domcke, W.; Opalka, D.; Poluyanov, L. V. Relativistic Theory of the Jahn-Teller Effect: p-Orbital in Tetrahedral and Trigonal Systems. J. Chem. Phys. 2016, 144, 124101.

(42) Weike, T.; Eisfeld, W. Development of Multi-Mode Diabatic Spin-Orbit Models at Arbitrary Order. J. Chem. Phys. 2016, 144, 104108.

(43) Zeng, T.; Seidu, I. Revisiting the $(E+A) \otimes(e+a)$ Problems of Polyatomic Systems with Trigonal Symmetry: General Expansions of Their Vibronic Hamiltonians. Phys. Chem. Chem. Phys. 2017, 19, 11098-11110.

(44) Seidu, I.; Goel, P.; Wang, X.-G.; Chen, B.; Wang, X.-B.; Zeng, T. Vibronic Interaction in $\mathrm{CO}_{3}$ Photo-Detachment: Jahn-Teller Effects beyond Structural Distortion and General Formalisms for Vibronic Hamiltonians in Trigonal Symmetries. Phys. Chem. Chem. Phys. 2019, 21, 8679-8690.

(45) Hickman, R. J.; Lang, R. A.; Zeng, T. General formalism for vibronic Hamiltonians in tetragonal symmetry and beyond. Phys. Chem. Chem. Phys. 2018, 20, 12312-12322. 
(46) Zeng, T.; Hickman, R. J.; Kadri, A.; Seidu, I. General Formalism of Vibronic Hamiltonians for Tetrahedral and Octahedral Systems: Problems That Involve T, E States and $t, e$ Vibrations. J. Chem. Theory Comput. 2017, 13, 5004-5018.

(47) Lang, R. A.; Japahuge, A.; Zeng, T. General Formalism of Vibronic Hamiltonians for Tetrahedral and Octahedral Systems: Problems that Involve $A$-Type States and $a$-Type Vibrations. Chem. Phys. 2018, 515, 36-45.

(48) Wang, K.; Zeng, T. Hamiltonian Formalism of Spin-Orbit Jahn-Teller and PseudoJahn-Teller Problems in Trigonal and Tetragonal Symmetries. Phys. Chem. Chem. Phys. 2019, 21, 18939-18957.

(49) Applegate, B. E.; Miller, T. A.; Barckholtz, T. A. The Jahn-Teller and Related Effects in the Cyclopentadienyl Radical, Part I: The Ab Initio Calculation of Spectroscopically observable Parameters. J. Chem. Phys. 2001, 114, 4855-4868.

(50) Applegate, B. E.; Bezant, A. J.; Miller, T. A. The Jahn-Teller and Related Effects in the Cyclopentadienyl Radical, Part II: Vibrational Analysis of the $\tilde{A}^{2} A_{2}^{\prime \prime}-\tilde{X}^{2} E_{1}^{\prime \prime} . J$. Chem. Phys. 2001, 114, 4869-4882.

(51) Maeda, S.; Ohno, K.; Morokuma, K. Updated Branching Plane for Finding Conical Intersections without Coupling Derivative Vectors. J. Chem. Theory Comput. 2010, 6, $1538-1545$.

(52) Mukherjee, S.; Ravi, S.; Naskar, K.; Sardar, S.; Adhikari, S. A beyond BornOppenheimer treatment of $\mathrm{C}_{6} \mathrm{H}_{6}^{+}$radical cation for diabatic surfaces: Photoelectron spectra of its neutral analog using time-dependent discrete variable representation. $J$. Chem. Phys. 2021, 154, 094306.

(53) Cano, J.; Costa, R.; Alvarez, S.; Ruiz, E. Theoretical Study of the Magnetic Properties of an Mn12 Single-Molecule Magnet with a Loop Structure: The Role of the NextNearest Neighbor Interactions. J. Chem. Theory Comput. 2007, 3, 782-788. 
(54) Szakács, P.; Kocsis, D.; Surján, P. R. Jahn-Teller Distortion of Ionized and Excited Carbon Nanotubes. J. Chem. Phys. 2010, 132, 034309.

(55) Reddy, V. S.; Camacho, C.; Xia, J.; Jasti, R.; Irle, S. Quantum Dynamics Simulations Reveal Vibronic Effects on the Optical Properties of $[n]$ Cycloparaphenylenes. J. Chem. Theory Comput. 2014, 10, 4025-4036.

(56) Reddy, V. S.; Irle, S. Indirect Intersystem Crossing $\left(S_{1} \rightarrow T_{3} / T_{2} \rightarrow T_{1}\right)$ Promoted by the Jahn-Teller Effect in Cycloparaphenylenes. J. Chem. Theory Comput. 2017, 13, $4944-4949$.

(57) Herzberg, G.; Teller, E. Schwingungsstruktur der Elektronenübergänge bei mehratomigen Molekülen. Z. Phys. Chem. 1933, 21B, 410-446.

(58) Renner, R. Zur Theorie der Wechselwirkung zwischen Elektronen- und Kernbewegung bei dreiatomigen, stabförmigen Molekülen. Z. Phys. 1934, 92, 172-193.

(59) Ryabinkin, I. G.; Izmaylov, A. F. Geometric Phase Effects in Dynamics Near Conical Intersections: Symmetry Breaking and Spatial Localization Geometric Phase Effects in Dynamics Near Conical Intersections: Symmetry Breaking and Spatial Localization. Phys. Rev. Lett. 2013, 111, 220406.

(60) Ryabinkin, I. G.; Joubert-Doriol, L.; Izmaylov, A. F. Geometric Phase Effects in Nonadiabatic Dynamics near Conical Intersections. Acc. Chem. Res. 2017, 50, 1785-1793.

(61) Li, J.; Joubert-Doriol, L.; Izmaylov, A. F. Geometric phase effects in excited state dynamics through a conical intersection in large molecules: N-dimensional linear vibronic coupling model study. J. Chem. Phys. 2017, 147, 064106.

(62) Garcia-Fernandez, P.; Bersuker, I. B. Pseudo Jahn-Teller origin of bending distortions in Renner-Teller molecules and its spectroscopic implications. Int. J. Quantum Chem. 2012, 112, 3025-3032. 
(63) Lang, R. A.; Hickman, R. J.; Zeng, T. VHEGEN: A vibronic Hamiltonian expansion generator for trigonal and tetragonal polyatomic systems. Computer Physics Communications 2020, 247, 106946.

(64) Brown, J.; Carrington, T. Using an expanding nondirect product harmonic basis with an iterative eigensolver to compute vibrational energy levels with as many as seven atoms. The Journal of Chemical Physics 2016, 145, 144104.

(65) Worth, G. A.; Beck, M. H.; Jäckle, A.; Meyer, H.-D. The MCTDH Package, Version 8.2, (2000), University of Heidelberg, Heidelberg, Germany. H.-D. Meyer, Version 8.3 (2002), Version 8.4 (2007). O. Vendrell and H.-D. Meyer, Version 8.5 (2011). 


\section{For Table of Contents use only}

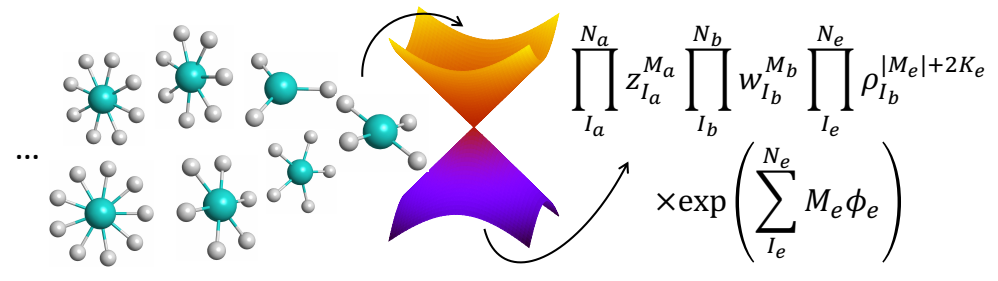

Table of Contents Graphic: Jahn-Teller and pseudo-Jahn-Teller problems in axial symmetries are funnelled to mathematical Hamiltonian expressions through the presented formalism. 